\title{
Cordyceps sinensis, un hongo usado en la medicina tradicional china
}

\author{
Carlos Illana Esteban
}

Departamento de. Biología Vegetal. Universidad de Alcalá, Alcalá de Henares, Madrid

Resumen Cordyceps sinensis (Berk.) Sacc. es un hongo ascomiceto conocido en China desde la antigüedad, que todavía se usa en la actualidad. Se hace un resumen de lo publicado con anterioridad en relación con este hongo: su hábitat, historia, comercialización y consumo, nomenclatura, composición farmacológica, cultivo y aplicaciones médicas.

Palabras clave Cordyceps sinensis, Chongcao, Medicina tradicional china

\section{Cordyceps sinensis, a fungi used in the Chinese traditional medicine}

Summary Cordyceps sinensis (Berk.) Sacc. is an ascomycete fungus known in China since antiquity, which is still being used today. A summary, showing relevant papers about this fungus, regarding habitat, history, marketing, consumption, nomenclature, pharmacological composition, culture and medical use, is presented.

Key words Cordyceps sinensis, Cordyceps mushroom, Caterpillar fungus, Chinese traditional medicine

\section{Introducción}

Cordyceps sinensis (Berk.) Sacc. es un hongo ascomiceto perteneciente a la familia Clavicipitaceae del orden Hypocreales, parásito de artrópodos, que ha sido altamente valorado en la medicina tradicional china durante siglos. Según el Diccionario de Hongos [9], hay 100 especies conocidas de Cordyceps, y la base de datos del Index Fungorum del CABI tiene 506 entradas para este género.

En China se conoce con los nombres de Hia tsao tong tchong o Dong Chong Xia Cao (chongcao) y en inglés como cordyceps mushroom o caterpillar fungus. No tiene nombre conocido en español al ser un hongo que no se encuentra en nuestro país y ser prácticamente desconocido.

Desde 1980, cerca de 2.000 artículos científicos se han publicado acerca de Cordyceps sinensis (1.500 de ellos en chino) [6]. En este trabajo se hace un resumen de lo publicado con anterioridad en relación con este hongo: su hábitat, historia, comercialización y consumo, nomenclatura, composición farmacológica, cultivo y aplicaciones médicas.

\section{Hábitat}

De modo natural, Cordyceps sinensis vive en la meseta de las altas montañas del Himalaya (3.000-5.000 m) en Tíbet, Nepal, India y algunas provincias de China. Este hábitat es compartido por las manadas de yaks que pastan en la alta montaña [7,14].

Es un hongo parásito que crece sobre las larvas de una mariposa nocturna conocida con el nombre de Chongcao bat (Hepialus armoricanus). Ocasionalmente se ha encontrado creciendo sobre larvas de otros insectos.

El micelio del hongo se desarrolla a partir de esporas existentes en el suelo. Durante el otoño se produce la infección de la oruga por parte del hongo, que penetra e invade su cuerpo, que permanece enterrado en el suelo. El hongo para su crecimiento absorbe todos sus nutrientes del cuerpo de la oruga. Hacia el verano del año siguiente, la infección provocada por el hongo ha matado a la oruga, y en su crecimiento ha consumido casi todo su cuerpo. El hongo que ha invadido el cuerpo del animal constituye un esclerocio y conserva la forma del cuerpo de la larva. De la cabeza de la oruga, que permanece enterrada en el suelo, surge una estructura erecta que emerge de la tierra y constituye el estroma [7,14]. El estroma, de unos $10 \mathrm{~cm}$ de alto, es de color azul oscuro a negro y contrasta con el verde de las altas praderas del Himalaya. Sobre el estroma se desarrollarán peritecios y, en su interior, ascosporas.

\section{Historia}

Cordyceps sinensis fue descubierto hace 1.500 años en el Tíbet por un pastor que observó cómo su ganado se volvía más vigoroso cuando comía este hongo. Las primeras referencias como hongo medicinal aparecen durante la
Dirección para correspondencia:

Dr. Carlos Illana Esteban

28871 Alcalá de Henares, Madrid, España

E-mail: carlos.illana@uah.es

(C2007 Revista Iberoamericana de Micología

Apdo. 699, E-48080 Bilbao (Spain)

$1130-1406 / 01 / 10.00 €$ 
dinastía Qing en China, en el tratado Ben-Cao-Cong-Xin (Nueva compilación de Materia Médica) de Wu-Yiluo en $1751[12,14,17]$.

Uno de los primeros occidentales en mencionar a este hongo con el nombre de hia tsao tong tchong fue el jesuita e historiador francés Du Halde [1]. En el tomo tres de su obra Description géographique, historique, chronologique, politique et physique Tartarie chinoise, describe cómo prepararlo para su consumo: "Il fault, me dit-il, prendre cinq drachmes ( 1 dracma $1,7 \mathrm{~g}$ ) de cette racine toute entiere avec fa queuë, et en farcir le ventre d'un canard domestique que vous ferez cuire à petit feu. Quand il fera cuit, retirez-en la drogue, dont la vertu aura paffé dans la chair du canard, et mangez-en foir et matin pendant huit ou dix jours“. Incluso Du Halde experimentó en sí mismo los efectos del consumo de Cordyceps sinensis [7].

En 1841 el hongo fue presentado en la London Entomological Society de esta manera: "Its a Chinese larva, from the back of the neck of each of which a slender fungus, twice as long as the body of the insect, had been produced. This insect, when thus attacked, is esteemed of great efficacy as a drug in China, where, from its very great rarity, it is only used by the emperor's physicians" [16].

En 1843 fue descrito e ilustrado formalmente por Berkeley con el nombre Sphaeria sinensis Berk. (epíteto considerado actualmente un sinónimo). Unos años más tarde, Saccardo lo transfirió definitivamente al género Cordyceps.

En 1918 C.G. Lloyd [2] publica las notas que aporta un corresponsal sobre el consumo de Cordyceps sinensis en China: "As a medicine it has a number and variety of uses. Boiled with pork it is employed as an antidote for opium poisoning and as a cure for opium eating. Also with pork and chicken it is taken as a tonic and a mild stimulant by convalescent persons and rapidly restores them to health and strength".

Los encargados de introducir Cordyceps sinensis en Estados Unidos fueron los hermanos Lloyd de Cincinnati [4]. Los hermanos Lloyd establecieron un negocio farmacéutico (Lloyd Brothers Manufacturing Pharmacists) a través del cual, en 1884, manufacturaron 835 productos. Su compañía sería comprada en 1960 por un farmaceútico alemán que llamó a la compañía Hoechst. Uno de los hermanos, Curtis Gates Lloyd, llegaría a ser un importante micólogo, especialista en Gasteromycetes.

\section{Comercialización y consumo}

Los circuitos de comercialización del Cordyceps sinensis recogido en la naturaleza son prácticamente secretos.

En China, en 1994, un kilogramo de hongo recogido en el campo podía alcanzar los 700 \$, y en el mercado internacional venderse a un precio que oscilaba entre 20.000-40.000 \$. Sharma [14] ha realizado un estudio sobre la comercialización de Cordyceps sinensis en el valle del río Gori Ganga en Nepal. En esta zona, en 2002, cerca de 900 personas lograron recolectar $186 \mathrm{~kg}$ del hongo, y entre los años 2000-2002 el precio de Cordyceps pagado al recolector ha aumentado más de cuatro veces.

Las orugas infectadas por el hongo se venden en pequeños bloques de unas 10 larvas. Al consumir el Cordyceps sinensis silvestre, se ingiere el estroma del hongo junto al cuerpo de la oruga, que ha sido invadido por el micelio [7].

El interés creciente por el Cordyceps sinensis natural ha llevado a los conservacionistas a considerar la sos- tenibilidad de este recurso, ya que no existe ninguna legislación sobre su recolección. La declaración de alguna de las zonas montañosas de Nepal, donde crece el hongo, como reserva de la biosfera no es la solución al problema, debido a los pocos recursos económicos con que cuentan las autoridades [14].

Con la apertura de China a partir de 1970, son muchas las personas de diferentes países que se han interesado por la medicina tradicional china. La gran demanda de Cordyceps y el elevado precio que alcanzan los ejemplares recolectados en la naturaleza, ha llevado a muchos comerciantes y distribuidores a adulterar las muestras, incluso las recogidas en el campo, en las que se insertan alambres de acero para que pesen más.

Actualmente lo que se comercializa como Cordyceps sinensis es, en muchas ocasiones, otro hongo. Se conocen más de 350 sustitutos, siendo el más empleado Cordyceps militaris (L.) Link [11]. Para evitar el fraude, sobre todo con otras especies de Cordyceps, se han propuesto distintos procedimientos y marcadores para ser usados en el control de calidad [5,14]. Como marcadores se pueden emplear nucleósidos, ergosterol, manitol y polisacáridos [10].

El consumo de Cordyceps sinensis puede hacerse simplemente hirviendo agua y echando el hongo como si se hiciera una sopa, o puede ser preparado con pollo, pato o cerdo. Para obtener sus efectos beneficiosos se recomienda tomar una dosis de 3-9 g al día [7].

\section{Composición farmacológica}

Los beneficios de tomar Cordyceps sinensis se encuentran en sus componentes químicos únicos: ácido cordicepídico, ácido glutámico, aminoácidos, poliaminas, D-manitol, esteroles, ácidos orgánicos, nucleósidos, vitaminas del grupo B y hasta 20 minerales [14,17,18]. Sin embargo, el conocimiento que se tiene sobre los componentes farmacológicos es todavía incompleto, debido a la variabilidad que existe en la composición, dependiendo de si el hongo se ha recolectado en el campo o se ha cultivado en el laboratorio, y en este último caso los componentes pueden ser distintos dependiendo de la cepa que se haya escogido. Para conocer la composición química completa de Hirsutella sinensis, anamorfo de Cordyceps sinensis, recomendamos el trabajo de Li et al. (2006) [11].

Uno de los nucleósidos es la 2',3' dideoxiadenosina (didanosina) que se comercializa con el nombre de Videx ${ }^{\circledR}$. La didanosina bloquea la enzima transcriptasa inversa y es usada como parte del tratamiento antirretroviral en pacientes con el virus VIH.

\section{Cultivo de Cordyceps}

La escasez de Cordyceps en la naturaleza ha elevado su precio en el mercado. Algunos investigadores han intentado solucionar la alta demanda del hongo cultivándolo en laboratorio. En 1982 el Instituto de Materia Médica de la Academia China de las Ciencias, logró aislar una cepa de Cordyceps sinensis, denominada Cs-4 y desarrolló un método de fermentación para producirla a nivel industrial. Cs-4 es el nombre comercial de la fase asexual de una de las cepas de Cordyceps sinensis, y es conocida por el nombre en latín de Paecilomyces hepiali. Cs-4 se comercializa en China a partir de 1980 con el nombre de cápsulas JinShuibao (JSBC).

Usado en más de 2.000 pacientes, los estudios clínicos realizados en China han demostrado que Cs-4 es 
muy efectivo y seguro, y muy similar al Cordyceps sinensis producido de forma natural.

Se han aislado otras cepas diferentes de Cordyceps sinensis. Han sido descritas 22 formas asexuales asociadas con el anamorfo de Cordyceps sinensis. Hirsutella sinensis Liu et al. ha sido considerado el anamorfo correcto [6].

Actualmente se emplean dos métodos para el cultivo de Cordyceps [4,5]. Uno u otro es usado por las empresas dependiendo de lo que quieran obtener en el producto final:

- Fermentación en medio líquido. Es el más usado en China. Consiste en que el hongo es introducido dentro de un tanque estéril con un medio líquido compuesto por residuos de gusanos de seda, al que se añaden carbohidratos y minerales. Utilizando este método el contenido de componentes bioactivos en el micelio es menor.

- Cultivo en sustrato sólido. Es el más empleado en Japón y América. Con este método el micelio se deja crecer en el interior de bolsas o frascos de plástico rellenos de un medio estéril, generalmente granos de cereal (arroz, trigo, centeno, mijo).

La composición cuantitativa y cualitativa del hongo es diferente según se emplee un método u otro. Cordyceps es químicamente muy complejo, y este cambio en la composición puede deberse a factores tan variables como el tipo de cepa empleada o la composición del medio de cultivo. Es difícil responder cuál de los dos métodos es el mejor, pero lo cierto es que la naturaleza es imposible de copiar.

Al comparar la composición de distintas cepas con el Cordyceps natural, se ha demostrado que estas no son coincidentes en ningún caso [5]. Algunos investigadores, con objeto de incrementar el contenido y cantidad de los componentes de Cordyceps, han procedido a hibridar dos cepas distintas del hongo en un medio con agar, al que se ha añadido veneno de serpiente de cascabel (Crotalus atrox), que debilita las paredes celulares. Esto permite el intercambio de material nuclear entre ambas cepas y la formación del híbrido, del que son analizados sus componentes activos.

\section{Aplicaciones médicas}

Los herboristas chinos comparan las cualidades de Cordyceps sinensis con el ginseng (Panax quinquefolius L.). El principal órgano de actuación en el organismo según la medicina tradicional china son los riñones.

Los usos terapéuticos de Cordyceps en la medicina tradicional china y en la actualidad han sido ampliamente investigados en Oriente. Se ha empleado con éxito en estudios con animales y en tratamientos clínicos con personas para tratar trastornos respiratorios, renales, hepáticos y cardiovasculares. Se ha demostrado asimismo su eficacia en tratamientos antitumorales. El consumo de Cs-4 disminuye los problemas de fatiga, además de aumentar la libido $[4,7,14,15]$.

Recientes investigaciones han confirmado que el uso de Cordyceps incrementa los niveles de ATP en la célula e incrementa la utilización de oxígeno. Eso explicaría que en 1993, durante la celebración de una competición nacional de atletismo en China, algunas atletas alcanzaron nuevos récords del mundo en las pruebas de 1.500, 3.000 y $10.000 \mathrm{~m}$, no demostrando síntomas de fatiga. La obtención de tan buenas marcas se debió, en parte, a un régimen especial en el que se incluía el consumo de Cordyceps sinensis [16].

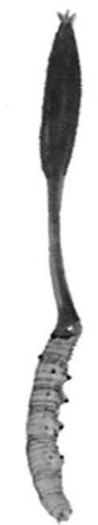

Figura 1. Estroma de Cordyceps sinensis emergiendo de la larva de Hepialus armoricanus, dentro de la cual crece el hongo.

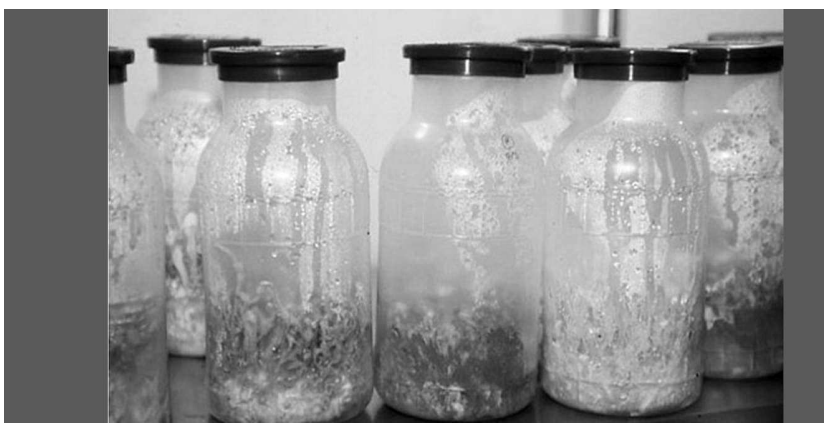

Figura 2. Cultivo en laboratorio de Cordyceps militaris (cortesía de Mushworld).
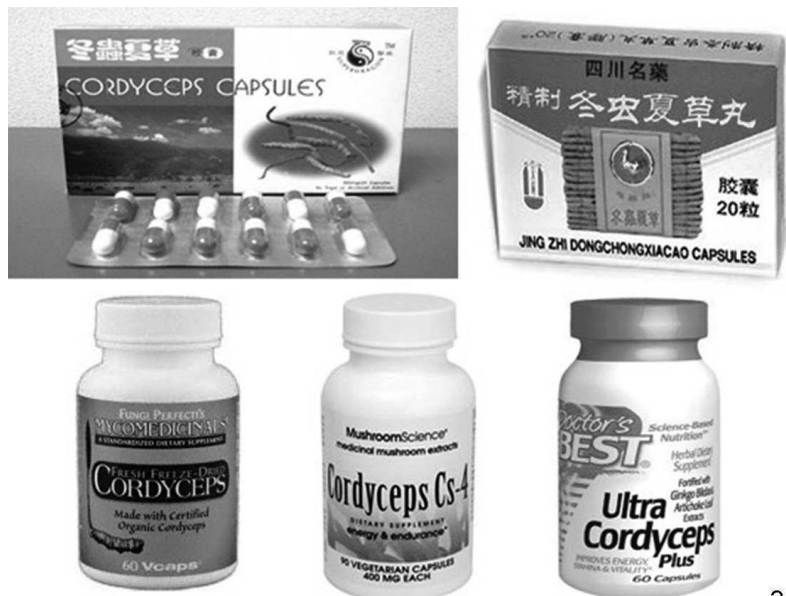

Figura 3. Comercialización de Cordyceps sinensis

\section{Otros fármacos obtenidos de Cordyceps}

La ciclosporina es una droga ampliamente usada en medicina. Actúa como un inmunosupresor en los trasplantes de órganos, para reducir el riesgo de rechazo. Es producida por un hongo que se aisló en el suelo en Noruega, en 1976. El hongo fue denominado Tolypocladium inflatum W. Gams. La ciclosporina ha sido probada para tratar otro tipo de enfermedades autoinmunes. En 1996 se descubrió que Tolypocladium inflatum es el estado asexual de otro hongo del género Cordyceps: Cordyceps subsessilis Petch [3]. 
Otro nuevo compuesto inmunosupresor ha sido aislado del hongo parásito de artrópodos Isaria sinclairii (Berk.) Lloyd (basiónimo Cordyceps sinclairii Berk.). El compuesto fue aislado por un grupo de investigación japonés en 1994 y fue denominado FTY720. Está siendo investigado por personal del Transplantation and Immunology Department del Novartis Institutes for BioMedical Research (NIBR) de Suiza [13]. Este compuesto es denominado "fingolimod" y aspira a convertirse en el mejor tratamiento para la esclerosis múltiple. Actualmente, fingolimod se encuentra en la fase III de los ensayos clíni$\cos [8]$.
El nucleósido conocido como "cordicepina" (3'-deoxiadenosina) fue aislado de Cordyceps militaris, pero es escaso o no se encuentra en Cordyceps sinensis [11]. La cordicepina ha resultado ser una sustancia anticancerígena empleada en medicina.
El autor agradece a MushWorld la autorización de la publicación de la figura 2.

\section{Bibliografia}

1. Du Halde JB. Description géographique, historique, chronologique, politique et historique, chronologique, politique
physique Tartarie chinoise. París, PG Lemercier, 1735.

2. Gist Gee N. Notes on Cordyceps sinensis. Myco Notes Lloyd Libr Mus 1918; 54: 767-768.

3. Hodge KT, Krasnoff SB, Humber RA. Tolypocladium inflatum is the anamorph of Cordyceps subsessilis. Mycologia 1996 88: 715-719.

4. Holliday J, Cleaver M. On the trail of the Yak. Ancient Cordyceps in the modern Yak. Ancient Cordyceps in the modern world (publicación electrónica). 2004. botanicals.org/)

5. Holliday JC, Cleaver P, Loomis-Powers M, Patel D. Analysis of quality and techniques for hybridization of medicinal fungus Cordyceps sinensis (Berk.) Sacc.

(Ascomycetes). Int J Med Mushroom 2004; 6: 147-160.

6. Jiang Y, Yao YJ. Names related to Cordyceps sinensis anamorph, Mycotaxon 2002; 84: 245-254.

7. Jones K. Cordyceps. Tonic food of Ancient China. Washington, Sylvan Press, 1997.
8. Kappos L, Antel J, Comi G, Montalban X O'Connor P, Polman CH, Haas T, Korn AA, Karsson G, Radue EW. Ora Korn AA, Karsson G, Radue EW. Oral
fingolimod (FTY720) for relapsing multiple fingolimod (FTY720) for relapsing multiple
sclerosis. New England J Med 2006; 355:

9. Kirk PM, Cannon PF, David JC, Stalpers JA. Ainsworth \& Bisby's Dictionary of the Fungi $\left(9^{\text {th }} \mathrm{ed}\right)$. Wallingford, CAB International, 2001.

10. Li SP, Yang GQ, Tsim KWK. Quality control of Cordyceps sinensis, a valued traditional Chinese medicine. J Pharm Biomed Analysis 2006; 41: 1571-1584.

11. Li C, Li Z, Fan M, Cheng W, Long Y, Ding T, Ming L. The composition of Hirsutella sinensis, anamorph of Cordyceps sinensis. J. Food Composition and Analysis 2006; 19: 800-805.

12. Pegler DN, Yao YJ, Li Y. The Chinese "caterpillar fungus". Mycologist 1994; 8 . 3-5.

13. Powell K. From ancient remedy to modern molecule, FTY720 offers new avenues for treating transplants and autoimmune disorders. Novartis Institutes for Biomedical Research (Nibr), summer 2004.
14. Sharma S. Trade of Cordyceps sinensis from high altitudes of the Indian from high altitudes of the Indian biotechnological priorities. Current biotechnological priorities. Current
Science 2004; 86: 1614-1619.

15. Sheng-Yuan W, Ming-Shi S. Pharmacological functions of Chinese medicinal fungus Cordyceps sinensis and realated species. J Food Drug Analysis 2000; 4: 248-257.

16. Steinkraus DC \& Whitfield JB. Chinese caterpillar fungus and world record runners. Amer Entomol 1994; 40: 235-238.

17. Zhu J, Halpern GM, Jones K. The scientific rediscovery of an Ancient Chinese Herbal Medicine: Cordyceps sinensis. Part I. J Altern Complement Med 1998; 4: 289-303.

18. Zhu JS, Halpern GM, Jones K. The scientific rediscovery of an Ancient
Chinese Herbal Medicine: Cordyceps sinensis. Part II. J Altern Complement sinensis. Part II. Altern 